\title{
In-Situ Synthesis of Flexible Nanocellulose/Carbon Nanotube/Polypyrrole Hydrogels for High- Performance Solid-State Supercapacitors
}

\section{Yang Zhan}

Wuhan University of Technology

\section{Yang Hu}

Wuhan University of Technology

\section{Yu Chen}

Wuhan University of Technology

Quanling Yang ( $\sim$ yangql@whut.edu.cn )

Wuhan University of Technology https://orcid.org/0000-0002-7013-419X

\section{Zhuqun Shi}

Wuhan University of Technology

\section{Chuanxi Xiong}

Wuhan University of Technology

\section{Research Article}

Keywords: Nanocellulose, Carbon nanotubes, Polypyrrole, Solid-state supercapacitor

Posted Date: February 15th, 2021

DOl: https://doi.org/10.21203/rs.3.rs-184104/v1

License: (1) This work is licensed under a Creative Commons Attribution 4.0 International License. Read Full License 


\section{Abstract}

Nanocellulose has become one of the most attractive matrix materials for flexible supercapacitors, owing to the high surface area, good mechanical properties and environmental friendliness. Herein, we developed electrode materials with high capacitance and mechanical flexibility through the in-situ synthesis of polypyrrole (PPy) in TEMPO-oxidized cellulose nanofibril (TOCN)/sulfonated carbon nanotubes (SCNT) composite hydrogels. The TOCN/SCNT/PPy composite hydrogels were thus obtained via a bifunctional $\mathrm{Fe}^{3+}$ in-situ oxidation, showing high specific capacitance of $5299 \mathrm{mF} / \mathrm{cm}^{2}$ at a current density of $1 \mathrm{~mA} / \mathrm{cm}^{2}$. Furthermore, the assembled symmetric TOCN-40SCNT-PPy solid-state supercapacitor exhibited outstanding capacitance of $375 \mathrm{mF} / \mathrm{cm}^{2}$ and electrochemical stability with $163.2 \%$ capacitance retention at a current density of $1 \mathrm{~mA} / \mathrm{cm}^{2}$ for 2500 cycles. These nanocellulose/carbon nanotube/polypyrrole hydrogels are thus promising in the fields of flexible solidstate supercapacitor with superior electrochemical performance.

\section{Introduction}

As a new type of energy storage device, supercapacitors have received extensive attention due to their unique power density and energy storage density characteristics differing from traditional storage devices. Supercapacitors have high power density, fast charge/discharge and ultralong cycling stability (Hou et al. 2010; Yu et al. 2011), which may bring some innovative products to electronic industrial equipment and mobile electronic devices. Considering the fact that electrode materials and dielectric materials in supercapacitors can be replaced by flexible and bendable soft materials, the application of supercapacitors in the field of mobile electronic devices (such as electronic skin) have great application prospects.

Cellulose is a natural polymer material, which could be used as a structurally reinforced flexible substrate or backbone in supercapacitors (Li et al. 2016; Li et al. 2014; Wang et al. 2019). 2,2,6,6tetramethylpiperidine-1-oxyl (TEMPO) oxidized cellulose nanofibrils (TOCN) obtained in the TEMPO/ $\mathrm{NaBr} / \mathrm{NaClO}$ aqueous system exhibit excellent performance, such as complete dispersion in water, small uniform diameter ( $3 \mathrm{~nm}$ ), high aspect ratio (greater than 200) and good mechanical properties (Isogai et al. 2011; Saito and Isogai 2014; Yang et al. 2018; Yang et al. 2017; Yin et al. 2020). Meanwhile, it was found that the abundant carboxylate groups on the surface of nanofibrils can promote the construction of TOCN-based functional nanocomposites (Wu et al. 2020; Xu et al. 2019; Xu et al. 2020; Yang et al. 2018).

Nanocellulose does not have high capacitance, so it is necessary to introduce other nanoscale electrode materials with high capacitance, mechanical flexibility and foldability (such as conductive polymers (ECP) (Liu et al. 2019; Hughes et al. 2004; Lota, Khomenko and Frackowiak 2004; Izadi-Najafabadi et al., 2001), metal oxides (Hiraoka et al. 2010; Snook et al. 2011; Wang et al. 2019), carbon materials (Lota et al. 2004; Hughes et al. 2004; Li et al. 2009), etc.). Dispersing these nanophase electrode materials uniformly in the TOCN network can provide extended ion transmission channels and facilitate ion 
transmission, which provides the supercapacitor with excellent charging and discharging rates. In addition, this also can reduce the volume change of electrode materials in many pseudocapacitive reactions. In order to eliminate the influence of internal collapse and performance degradation caused by the volume changes, (Naoi and Simon 2008; Yan et al. 2014) (for example, the redox reaction of conductive polymers involves the doping and dedoping of counter ions), cellulose and electrode materials were could compounded together to improve the cycling stability of supercapacitors.

Carbon nanotubes (CNTs) have the advantages of low density, regular 1D tubular structure, good mechanical properties, excellent electrochemical stability and high specific surface area, thus they are widely used in the preparation of supercapacitor electrode materials (Shao et al. 2015). However, the hydrophobicity of CNTs makes it very easy to aggregate, resulting in inability to uniformly disperse CNTs. Thus, excellent electrochemical performance cannot be fully realized. Currently, grafting surfactants or strong acid modification methods on the surface of CNTs are usual to improve the dispersion properties of CNTs (Zang et al. 2015; Zhan et al. 2019; Zhao et al. 2010). However, supercapacitors made from pure carbon materials are generally lower in specific capacitance. As a result, researchers have shifted the focus from pure carbon materials to pseudocapacitive materials (such as transition metal oxides/hydroxides or conductive polymers) which have a specific capacitance that is $3-7$ times greater than the former (Merlet et al. 2012). Polypyrrole (PPy) is the most studied conductive polymer used in energy storage applications. The monomer is light in weight, and the theoretical specific capacity at a doping ratio of $25 \%$ is $100 \mathrm{mAh} / \mathrm{g}$ (Naoi and Simon 2008). PPy obtained by chemical or electrochemical polymerization is combined with various substrates to prepare a conductive polymer-based electrode material (Novák et al. 1997).

Herein, a novel hybrid flexible supercapacitor is design and fabricated. The formation of TOCN/SCNT/PPy hydrogel used the TOCN as the matrix to construct the 3D porous framework, sulfonated carbon nanotubes (SCNT) as the filler and the situ synthesis of PPy in the substrate. The hybrid hydrogel was formed by crosslinking of ferric salt and polymerization of pyrrole through ferric salts too (oxidative initiation). The negative charges carried on the surface of SCNTs grafted with paminobenzenesulfonic acid electrostatically repel each other, so that they can be uniformly dispersed in water, hindering the agglomeration of CNTs. The TOCN/SCNT/PPy hydrogel presents notable electrochemical performance, and the assembled TOCN/SCNT/PPy aerogel-based all-solid supercapacitor possesses an excellent capacitance retention.

\section{Experimental}

\section{Materials}

Softwood bleached kraft pulp (SBKP) was from Nippon Paper Industries (Tokyo, Japan). NaClO was bought from Aladdin (Shanghai, China). TEMPO and $\mathrm{NaNO}_{2}$ were purchased from Sigma-Aldrich Corporation (Saint Louis, USA). Multiwalled carbon nanotubes (MCNTs) were purchased from Shenzhen 
Nano Co., Ltd (China). Pyrrole, $\mathrm{NaBr}, \mathrm{NaOH}$, and other chemical reagents were of analytical grade and obtained from Sinopharm Chemical Reagent Co. Ltd (China).

\section{Preparation of TOCN dispersion}

TOCN was prepared from SBKP by the TEMPO-oxidized method (Isogai et al., 2011; Wu et al., 2020). In brief, $0.2 \mathrm{~g} \mathrm{NaBr}, 0.032 \mathrm{~g}$ TEMPO and $200 \mathrm{~g}$ deionized water were added into a beaker. Then wet SBKP (water content $80 \%$, dry weight $2 \mathrm{~g}$ ) was dispersed in the mixture under stirring, and $5.65 \mathrm{ml} \mathrm{NaClO}$ solution $(1.77 \mathrm{~mol} / \mathrm{L})$ was added to the suspension. The $\mathrm{pH}$ was adjusted to 10 by adding $0.5 \mathrm{M} \mathrm{NaOH}$. The obtained TEMPO-oxidized pulp was washed with water. Then it was re-dispersed in deionized water, sonicated and centrifuged to obtain the TOCN dispersion.

\section{Preparation of sulfonated carbon nanotubes}

$20 \mathrm{ml}$ of a $1 \mathrm{~mol} / \mathrm{L}$ sodium nitrite solution was added to a mixed solution of $20 \mathrm{ml}$ of $5 \mathrm{wt} \% \mathrm{NaOH}$ solution and $3.4 \mathrm{~g}$ of $\mathrm{p}$-aminobenzenesulfonic acid. Then, the above solution was transferred to an ice water bath and $1 \mathrm{~mol} / \mathrm{L}$ hydrochloric acid was added. When the solution became yellowish, a diazonium salt solution was obtained. Subsequently, $0.4 \mathrm{~g}$ of carbon nanotubes were added to the diazonium salt solution prepared above, stirred for $24 \mathrm{~h}$ and washed three times with deionized water until the filtrate was colorless. Finally, the products on the filter paper were dried in a vacuum oven at $60{ }^{\circ} \mathrm{C}$ to obtain sulfonation carbon nanotubes and coded as SCNTs.

\section{Preparation of TOCN/SCNT/PPy hydrogel working electrodes}

Different amounts of SCNTs were added to the $0.5 \mathrm{wt} \%$ TOCN dispersion followed by stirring for $1 \mathrm{~h}$, sonicating for 4 minutes and removing the air bubbles. Then, the above mixture was poured into a plastic mold inserted with carbon cloth, and $1 \mathrm{ml}$ of $0.5 \mathrm{~mol} / \mathrm{L} \mathrm{Fe}(\mathrm{NO})_{3}$ solution was added and allowed to stand for $12 \mathrm{~h}$ to obtain the TOCN/SCNT hydrogels, which were denoted as TOCN-40SCNT and TOCN-50SCNT with the SCNT content of $40 \mathrm{wt} \%$ and $50 \mathrm{wt} \%$, respectively. Finally, the prepared TOCN/SCNT hydrogels were placed in a pyrrole atmosphere for $12 \mathrm{~h}$ to obtain TOCN/SCNT/PPy hydrogels, which were labeled as TOCN-40SCNT-PPy and TOCN-50SCNT-PPy. In this process, pyrrole was polymerized on the surface of nanocellulose under the action of $\mathrm{Fe}^{3+}$. The TOCN/SCNT/PPy composite hydrogels prepared above were then cut into thickness of $1 \mathrm{~mm}$ and area of $1 \times 1 \mathrm{~cm}^{2}$. They were directly placed in $1 \mathrm{~mol} / \mathrm{L} \mathrm{H}_{2} \mathrm{SO}_{4}$ electrolyte (in a three-electrode system) for electrochemical testing.

\section{Fabrication of TOCN/SCNT/PPy aerogel based solid-state supercapacitor in two-electrode system}

The water in the above obtained TOCN-40SCNT-PPy hydrogels was replaced by ethanol and $t$-butanol. After the solvent was completely replaced, the alcohol gels were frozen in liquid nitrogen, and freeze-dried for $48 \mathrm{~h}$ to obtain TOCN-40SCNT-PPy aerogels. The TOCN-40SCNT-PPy aerogels prepared above were pressed into composite aerogel films of $10 \mathrm{~mm} \times 10 \mathrm{~mm}$ under a pressure of $1 \mathrm{MPa}$. Then the aerogel 
films were immersed into the polyvinyl alcohol (PVA) $/ \mathrm{H}_{2} \mathrm{SO}_{4}$ gel electrolyte $\left(6 \mathrm{~g}\right.$ PVA and $6 \mathrm{~g} \mathrm{H}_{2} \mathrm{SO}_{4}$ was dissolved into $60 \mathrm{~mL}$ deionized water at $85^{\circ} \mathrm{C}$ ) for $12 \mathrm{~h}$ as the working electrode.

\section{Analysis}

The peak of each functional group in the composite was observed in the infrared spectrum measured by the Fourier infrared spectrometer (Nexus, USA). The crystal structure of the composite was analyzed by Xray powder diffractometry (XRD) (D8 Advance). The chemical environment of carbon and nitrogen in the composite was analyzed and tested by X-ray photoelectron spectroscopy (XPS) (V G Multilab 2000). The cross section of the aerogel was scanned with a field emission scanning electron microscope (SEM) (Hitachi S-4800), and the cross section was obtained by freezing and brittle fracture with liquid nitrogen. The polypyrrole content in the composite gel can be calculated from the nitrogen content measured by the elemental analyzer (EA) (CHNS). The specific surface area and porosity of the solid supercapacitor were tested on the sample with the specific surface area and porosity analyzer (BET) (ASAP 2020M) with the adsorbed gas as $\mathrm{N}_{2}$ and temperature as $40^{\circ} \mathrm{C}$.

\section{Electrochemical Measurements}

The electrochemical properties were measured by cyclic voltammetry (CV), galvanostatic charge/discharge (GCD), and electrochemical impedance spectroscopy (EIS) tests by $\mathrm{CHI} 660 \mathrm{E}$. The areal capacitance $\left(C_{A}, \mathrm{mF} \mathrm{cm}^{-2}\right)$, energy density $\left(\mathrm{Ea}, \mu \mathrm{W} \mathrm{h} \mathrm{cm}{ }^{-2}\right)$ and power density $\left(\mathrm{Pa}, \mu \mathrm{W} \mathrm{cm}^{-2}\right)$ were calculated by GCD test using the following equation:

$$
\begin{gathered}
C_{a}=\frac{I \times \Delta t}{S \times \Delta U} \\
E_{a}=\frac{C_{a} \times \Delta U^{2}}{2 \times 3.6} \\
P_{a}=\frac{E_{a} \times 3600}{\Delta t}
\end{gathered}
$$

where / is the charge-discharge current, $\Delta t$ is the discharge time, $S$ is the electrode area, $\Delta U$ is the potential window subtracting IR.

\section{Results And Discussion}

\section{Characterization of TOCN/SCNT/PPy}

Fig. 1 and Fig. S1 show the FT-IR spectra of TOCN, SCNT, PPy, TOCN/SCNT and TOCN/SCNT/PPy aerogels with different filler loadings. The TOCN/SCNT/PPy aerogels exhibited two characteristic bands at 1613 and $1034 \mathrm{~cm}^{-1}$, which belong to the -COO group of TOCN and S-O stretching vibration peak of the aromatic sulfonic acid of SCNT, respectively. The antisymmetric ring stretching mode absorption peaks of PPy at 1460 and $1550 \mathrm{~cm}^{-1}$ shifted to 1455 and $1548 \mathrm{~cm}^{-1}$ in the composite aerogels, respectively, indicating the formation of hydrogen bonds between the hydroxyl groups of TOCN and the N-H groups of pyrrole rings. 
Fig. 2 and Fig. S2 show XRD patterns of TOCN, SCNT, TOCN/SCNT and TOCN/SCNT/PPy aerogels. TOCN aerogel exhibits typical peaks at $14^{\circ}, 16^{\circ}$, and $23^{\circ}$, corresponding to the (110) and (200) crystal planes of native cellulose I. SCNT powder shows two diffraction peak at $26^{\circ}$ and $44^{\circ}$ owing to (002) and (100) crystal planes of carbon nanotubes. It reveals that the natural cellulose crystal structure of TOCN was preserved in the process of TEMPO oxidation and SCNT could maintain the crystal structure of carbon nanotubes well. The TOCN/SCNT/PPy composite aerogels possessed partial characteristic diffraction peaks of TOCN and SCNT, the (100) crystal plane of SCNT were not obvious, and the diffraction peak at $26^{\circ}$ of SCNT was widened. It indicated that TOCN could hinder the agglomeration of SCNT to a certain extent.

To further study the detailed chemical structure, the element compositions of TOCN/SCNT/PPy were analyzed by XPS and shown in Fig. 3. The $\mathrm{C} 1 \mathrm{~s}$ peak is fitted into four peaks for TOCN-40SCNT-PPy, with the peaks at 284.6, 286.1, 287 and $287.7 \mathrm{eV}$ corresponding to $\mathrm{C}-\mathrm{C} / \mathrm{C}=\mathrm{C}, \mathrm{C}-\mathrm{O} / \mathrm{C}-\mathrm{N}, \mathrm{C}=\mathrm{O}$ and $\mathrm{O}-\mathrm{C}=\mathrm{O}$, respectively (Fig. 3a). These peaks are mainly derived from oxygen-containing functional groups on SCNTS and TOCN, which make the composites exhibit good hydrophilic properties and excellent rewetting property in an aqueous electrolyte, increasing the utilization rate of the electrochemically active material. Three different $\mathrm{N}$ bonds are obtained for TOCN-40SCNT-PPy from the N 1s spectrum (Fig. 3b), namely, -N$(398.2 \mathrm{eV}),-\mathrm{N}=(399.8 \mathrm{eV})$ and $-\mathrm{N}^{+}-(401.1 \mathrm{eV})$. It is indicated that PPy exists in nanocellulose-based gels, which is consistent with the results of FTIR.

\section{Microstructure of TOCN/SCNT/PPy}

SEM images of the TOCN-40SCNT-PPy and TOCN-50SCNT-PPy aerogels are shown in Fig. 4 to study their morphology. The TOCN/SCNT/PPy aerogels exhibited three-dimensional network structures, which were formed by random orientation and interconnection of one-dimensional nanofibrils, and rich pores were present between the nanofibrils. The fibrils were distinctly observed because TOCNs were entangled with the SCNTs, and a uniform layer of conductive PPy was deposited on the surface of TOCNs or SCNTs (Fig. 4a-d). Furthermore, it could be clearly observed from the TEM image in Fig. 4e that relatively thick SCNTs were present between TOCNs, and PPy was deposited on both surfaces to form a three-dimensional structure with conductivity. It is consistent with the results of SEM.

In order to further study the internal microstructure of the composite aerogels, nitrogen adsorption/desorption isotherms and Barrett-Joyner-Halenda (BJH) pore size distribution of TOCN40SCNT-PPy and TOCN-50SCNT-PPy aerogels were performed in Fig. 5. The specific surface area and pore volume of TOCN-40SCNT-PPy aerogel are $334 \mathrm{~m}^{2} / \mathrm{g}$ and $0.76 \mathrm{~cm}^{3} / \mathrm{g}$, respectively, indicating an abundant pore structure. When the content of SCNT in the electrode was increased, the shape of the nitrogen adsorption/desorption isotherm remained unchanged, indicating that the three-dimensional structure with pores was maintained inside. Compared with TOCN-40SCNT-PPy, TOCN-50SCNT-PPy possessed a lower specific surface area of $300 \mathrm{~m}^{2} / \mathrm{g}$ and a smaller pore volume of $0.71 \mathrm{~cm}^{3} / \mathrm{g}$. The decrease in specific surface area may be attributed to the fact that the specific gravity of the active material such as sulfonated carbon nanotubes and polypyrrole in the electrode were too large, and partial 
packing occurred to lower the theoretical specific surface area of the material (Fig. 5a). In addition, the pore sizes of the two different components aerogels were mainly distributed between 2-10 nm, reflecting that the aerogels mainly possessed mesoporous structures (Fig. 5b).

Table 1. Elemental analysis and PPy content of TOCN/SCNT/PPy aerogels.

\begin{tabular}{|llll|}
\hline Sample & $\mathrm{C}(\mathrm{wt} \%)$ & $\mathrm{N}(\mathrm{wt} \%)$ & $\mathrm{PPy}(\mathrm{wt} \%)$ \\
\hline TOCN-40SCNT-PPy & 59.40 & 3.84 & 17.55 \\
\hline TOCN-50SCNT-PPy & 67.44 & 2.98 & 14.28 \\
\hline
\end{tabular}

Elemental analysis and the relevant PPy content of TOCN/SCNT/PPy aerogel are shown in Table 1. It could be seen from the table that when the amount of SCNT was small (e.g. TOCN-40SCNT-PPy), the C content was relatively small. By calculating the mass fraction of $\mathrm{N}$, the content of polypyrrole in the TOCN-40SCNT-PPy aerogel was $17.55 \mathrm{wt} \%$, which was slightly higher than the content of polypyrrole in TOCN-50SCNT-PPy (i.e. $14.28 \mathrm{wt} \%$ ). Therefore, when the content of SCNT in cellulose is different, there is no significant influence on the content of PPy in the whole aerogel.

\section{Electrical characteristics of TOCN/SCNT/PPy}

Fig. 6 and Fig. S3 show the CV curves of TOCN/40SCNT, TOCN/50SCNT and TOCN-40SCNT-PPy and TOCN-50SCNT-PPy hydrogel-based supercapacitors with a potential window of -0.2-0.6 $\mathrm{V}$ and a scan rate of $20-100 \mathrm{mV} / \mathrm{s}$. It's observed that the TOCN/SCNT/PPy hydrogel-based supercapacitor exhibited a symmetrical, approximately rectangular-like CV curves. Compared with TOCN-50SCNT-PPy, TOCN40SCNT-PPy had almost no change in the shape of the curve as the scanning rate increased, and it exhibited a rectangular-like shape even at a large scanning rate of $100 \mathrm{mV} / \mathrm{s}$, indicating that the internal structure of TOCN-40SCNT-PPy was beneficial to the reversible diffusion of electrolyte ions and could work at high current. Therefore, the TOCN-40SCNT-PPy hydrogel-based supercapacitor exhibited better capacitance performance than the TOCN-50SCNT-PPy. In general, a suitable amount of active material facilitates the formation of a continuous conductive three-dimensional network in a cellulose-based supercapacitor. However, excessive active filler will form local accumulation, which causes a significant decrease in capacitance and conductivity.

Fig. 7 and Fig. S4 show the areal specific capacitances of various TOCN/SCNT/PPy hydrogels at different current densities estimated based on the GCD tests. At a current density of $1 \mathrm{~mA} / \mathrm{cm}^{2}$, the area specific capacitance of the TOCN-40SCNT-PPy hydrogel-based supercapacitor was $5299 \mathrm{mF} / \mathrm{cm}^{2}$, which was higher than in previously reported studies (Wu et al. 2020); while the area specific capacitance of TOCN-50SCNT-PPy at the same current density was $1781 \mathrm{mF} / \mathrm{cm}^{2}$ (Fig. 7b). Because the proportion of SCNT in TOCN-50SCNT-PPy was larger, the microstructure was denser and the diffusion of electrolyte ions inside the material became difficult. The TOCN-40SCNT-PPy hydrogel with an appropriate amount of 
active material had a thin active material layer, which improved the diffusion of the electrolyte, and could fully realize the electrochemical performance of the SCNT and PPy. In addition, as the charge-discharge current density increased, the area capacitance of the all electrodes decreased. This was due to the electrolyte ions in the depth of the pores didn't participate in the reaction under higher charge-discharge current density, and the capacity of electrolyte could not be fully expressed. When the current density was increased to nearly $10 \mathrm{~mA} / \mathrm{cm}^{2}$, the decay of the material capacity approached a plateau state, indicating that the effect of electrolyte ion diffusion had little effect on the area capacitance of the material (Fig. 7c). Additionally, TOCN-40SCNT-PPy hydrogel had a high energy density of up to $471 \mu \mathrm{Wh} / \mathrm{cm}^{2}$ at a power density of $568 \mu \mathrm{W} / \mathrm{cm}^{2}$ (Fig. S5)

Electrochemical impedance spectroscopy (EIS) was used to analyze the transport behavior of electrons and ions (Fig. 8). The equivalent series resistance of the TOCN/SCNT/PPy hydrogel supercapacitor was approximately $2 \Omega$, indicating that the interfacial impedance of the hydrogel was small. In the intermediate frequency region, the Warburg impedance of TOCN-50SCNT-PPy hydrogel was much lower than that of TOCN-40SCNT-PPy, because the pore structure of TOCN-40SCNT-PPy hydrogel was relatively loose, which is beneficial to the diffusion of electrolyte ions. In addition, in the low-frequency region, the Nyquist plot of TOCN-40SCNT-PPy approximates a vertical line, indicating a perfect capacitive behavior.

The cyclic stability of TOCN-40SCNT-PPy was investigated by consecutive GCD cycling at a current density of $5 \mathrm{~mA} \mathrm{~cm}^{-2}$ as shown in Fig. 9, which still had a capacitance retention of $82 \%$ over 2000 cycles. On the one hand, the flexible matrix of nanocellulose could inhibit the molecular chain damage of PPy caused by volume changes in the charge and discharge cycles; on the other hand, the good ratio of the three components promotes the formation of the conductive porous three-dimensional network structure. Therefore, the hydrogel has good cycle performance.

In order to explore the practical application of the TOCN/SCNT/PPy electrode for flexible supercapacitors, a symmetric all-solid-state supercapacitor was prepared. The electrochemical performance of the TOCN40SCNT-PPy solid-state supercapacitor was characterized in a two-electrode system with $\mathrm{PVA} / \mathrm{H}_{2} \mathrm{SO}_{4}$ as electrolyte and separator. The CV curves of the TOCN-40SCNT-PPy solid-state supercapacitor exhibited approximately rectangular-like shape, and it was well maintained even at $100 \mathrm{mV} / \mathrm{s}$ scan rate, indicating a good reversibility and quick charge-transfer capability (Fig. 10a). The TOCN-40SCNT-PPy solid-state supercapacitor possessed a high areal specific capacitance of $233 \mathrm{mF} / \mathrm{cm}^{2}$ at a current density of 0.5 $\mathrm{mA} / \mathrm{cm}^{2}$ (Fig. 10b). The carboxyl group on the surface of TOCN and the sulfonic acid group on the surface of SCNT are negatively charged, and electrostatic repulsion is generated to uniformly disperse both in water and hinder the formation of SCNTs. Using $\mathrm{Fe}^{3+}$ as a cross-linking agent, TOCN and SCNT were entangled by coordination. The addition of SCNT could improve the conductivity and stability of the network structure, but the specific capacitance was poor. Therefore, PPy was introduced into the network, which deposited on the surface of the TOCN through hydrogen bonding, further improving the overall conductivity and electrochemical properties of the material. 
The TOCN-40SCNT-PPy solid-state supercapacitor exhibited a steep oblique line in the low-frequency region in EIS curve, indicating the ideal capacitive response. The intercept of the curve at high frequency was $4 \Omega$, which represented the equivalent series resistance was about $4 \Omega$. And the smaller Warburg impedance was also observed in the intermediate frequency region, owing to the hydrophilicity of the TOCN and easy ion transmission in the robust porous network of TOCN-40SCNT-PPy gel (Fig. 10c). The electrochemical stability of the TOCN-40SCNT-PPy solid-state supercapacitor was tested at a current density of $1 \mathrm{~mA} / \mathrm{cm}^{2}$ for 2500 cycles (Fig 10d). It is noticeable that the capacitance of the TOCN-40SCNTPPy solid-state supercapacitor increased by $63.2 \%$ after 2500 charge/discharge cycles, and the maximum specific capacity in the cycles was up to $375 \mathrm{mF} / \mathrm{cm}^{2}$. The excellent cycling stability of the solid-state supercapacitor may be attributed to the superior 3D porous network structure and the PPy filled in a 3D network structure was gradually activated in the charge and discharge cycles.

\section{Conclusions}

In this work, we demonstrate an electrode material with high capacitance and mechanical flexibility for supercapacitors by in-situ synthesis of PPy in TOCN matrix and SCNT. A facile fabrication process was achieved, in which TOCN/SCNT/PPy hydrogels were prepared via a bifunctional $\mathrm{Fe}^{3+}$ in-situ oxidiation method and the hydrogels were converted to aerogels by solvent-exchanging and freeze-drying. The TOCN-40SCNT-PPy hydrogel exhibited a high specific capacitance of $5299 \mathrm{mF} / \mathrm{cm}^{2}$ at a current density of $1 \mathrm{~mA} / \mathrm{cm}^{2}$, probably resulting from a large surface area, 3D network porous structure and sufficient electrical conductivity of the composites. Furthermore, the assembled symmetric TOCN-40SCNT-PPy based solid-state supercapacitor exhibited outstanding capacitance of $375 \mathrm{mF} / \mathrm{cm}^{2}$ and electrochemical stability with $163.2 \%$ capacitance retention at a current density of $1 \mathrm{~mA} / \mathrm{cm}^{2}$ for 2500 cycles. This got benefit from the PPy covered on the surface of the TOCN-SCNT-PPy aerogel gradually activated in the charge and discharge cycles. The employing of the flexible nanocellulose substrate provides prominent opportunity by solving the problem of poor cycle stability of PPy based supercapacitors.

\section{Declarations}

\section{Associated content}

Supporting information Further details on FT-IR spectra, XRD patterns, CV curves, Galvanostatic chargedischarge (GCD) curves, Nyquist impedance spectra based on TOCN-40SCNT and TOCN-50SCNT hydrogels.

Acknowledgments This work was supported by the National Natural Science Foundation of China (No. 51703177 and 21704079), the Fundamental Research Funds for the Central Universities (WUT:

2020IB030) and Key laboratory of Processing and Quality Evaluation Technology of Green Plastics of China National Light Industry council, Beijing Technology and Business University, Beijing 100048, China (No. PQETGP2019007). 


\section{Compliance with ethical standards}

Conflict of interest The authors declare that they have no conflict of interest.

\section{References}

1. Hiraoka T, Izadi-Najafabadi A, Yamada T et al (2010). Compact and light supercapacitor electrodes from a surface-only solid by opened carbon nanotubes with $2200 \mathrm{~m}^{2} / \mathrm{g}$ surface area. Adv Funct Mater (20):422-428. https://doi.org/10.1002/adfm.200901927.

2. Hou Y, Cheng Y, Hobson T, Liu J (2010). Design and synthesis of hierarchical $\mathrm{MnO}_{2}$ nanospheres/carbon nanotubes/conducting polymer ternary composite for high performance electrochemical electrodes. Nano Lett 10(7):2727-2733. https://doi.org/10.1021/nl101723g.

3. Hughes M, Chen GZ, Shaffer MSP et al (2004). Controlling the nanostructure of electrochemically grown nanoporous composites of carbon nanotubes and conducting polymers. Compos Sci Technol 64:2325-2331. https://doi.org/10.1016/j.compscitech.2004.01.026.

4. Isogai A, Saito T, Fukuzumi H (2011). TEMPO-oxidized cellulose nanofibers. Nanoscale 3:71-85. https://doi.org/10.1039/c0nr00583e.

5. Izadi-Najafabadi A, Yasuda S, Kobashi, K et al (2001). Extracting the full potential of single-walled carbon nanotubes as durable supercapacitor electrodes operable at $4 \mathrm{~V}$ with high power and energy density. Adv Mater (22):E235-E241. https://doi.org/10.1002/adma.200904349.

6. Li H, Wang J, Chu Q et al (2009). Theoretical and experimental specific capacitance of polyaniline in sulfuric acid. J Power Sources 190 (2):578-586. https://doi.org/10.1016/j.jpowsour.2009.01.052.

7. Li S, Huang D, Yang J et al (2014). Freestanding bacterial cellulose-polypyrrole nanofibres paper electrodes for advanced energy storage devices. Nano Energy (9):309-317. https://doi.org/10.1016/j.nanoen.2014.08.004.

8. Li Z, Liu J, Jiang K, Thundat T (2016). Carbonized nanocellulose sustainably boosts the performance of activated carbon in ionic liquid supercapacitors. Nano Energy 25:161-169. https://doi.org/10.1016/j.nanoen.2016.04.036.

9. Liu Z, Chen J, Zhan Y et al (2019). Fe ${ }^{3+}$ crosslinked polyaniline/cellulose nanofibril hydrogels for high-performance flexible solid-state supercapacitors. ACS Sustain. Chem Eng 7(21):17653-17660. https://doi.org/10.1021/acssuschemeng.9b03674.

10. Lota K, Khomenko V, Frackowiak E (2004). Capacitance properties of poly(3,4ethylenedioxythiophene)/carbon nanotubes composites. J Phys Chem Solids 65:295-301. https://doi.org/10.1016/j.jpcs.2003.10.051.

11. Merlet C, Rotenberg B, Madden PA et al (2012). On the molecular origin of supercapacitance in nanoporous carbon electrodes. Nat Mater (11):306. https://doi.org/10.1038/nmat3260.

12. Naoi K, Simon P (2008). New Materials and new configurations for advanced electrochemical capacitors. Electrochem. Soc Interface (17):34-36. https://doi.org/10.1149/2.f04081if 
13. Novák P, Müller K, Santhanam KSV, Haas O (1997). Electrochemically active polymers for rechargeable batteries. Chem Rev (97):207. https://doi.org/10.1021/cr941181o.

14. Saito T, Isogai A (2014). TEMPO-mediated oxidation of native cellulose. The Effect of oxidation conditions on chemical and crystal structures of the water-insoluble fractions. Biomacromolecules 5:1983-1989. https://doi.org/10.1021/bm0497769.

15. Shao Q, Tang J, Lin Y et al (2015). Carbon nanotube spaced graphene aerogels with enhanced capacitance in aqueous and ionic liquid electrolytes. J Power Sources (278):751-759. https://doi.org/10.1016/j.jpowsour.2014.12.052.

16. Snook GA, Kao P, Best AS (2011). Conducting-polymer-based supercapacitor devices and electrodes. J Power Sources (196):1-12. https://doi.org/10.1016/j.jpowsour.2010.06.084.

17. Song Y, Shi Z, Hu G-H, Xiong C, Isogai A, Yang Q (2021) Recent advances in cellulose-based piezoelectric and triboelectric nanogenerators for energy harvesting: a review. J Mater Chem $A$. https://doi.org/10.1039/D0TA08642H

18. Wang C, Yang Z, Wang X, Yu Q (2019) New research progress of functional wood. J For Eng 4(03):10-18. https://doi.org/10.13360/j.issn.2096-1359.2019.03.002

19. Wang X, Li X, Ge W, Yang Y (2019) Progress in biomass-derived carbon materials/MnO2 composite and its application in supercapacitors. J For Eng 4(06):1-10. https://doi.org/10.13360/j.issn.20961359.2019.06.001

20. Wu H, Li W, Zhao M, Lu S, Huang L, Chen L (2020) Progress in cellulose-based self-healing gels. J For Eng 5(01):11-17. https://doi.org/10.13360/j.issn.2096-1359.201907031

21. Wu T, Song Y, Shi Z et al (2021). High-performance nanogenerators based on flexible cellulose nanofibril/MoS 2 nanosheet composite piezoelectric films for energy harvesting. Nano Energy 80 : 105541. https://doi.org/ 10.1016/j.nanoen.2020.105541.

22. Xu H, Liu Y, Xie Y, Zhu E, Shi Z, Yang Q, Xiong C (2019) Doubly cross-linked nanocellulose hydrogels with excellent mechanical properties. Cellulose 26:8645-8654. https://doi.org/10.1007/s10570-01902689-2

23. Xu H, Xie Y, Zhu E et al (2020). Supertough and ultrasensitive flexible electronic skin based on nanocellulose/sulfonated carbon nanotube hydrogel films. J Mater Chem A 8:6311-6318. https://doi.org/10.1039/d0ta00158a.

24. Yan J, Wang Q, Wei T, Fan ZJ (2014). Recent advances in design and fabrication of electrochemical supercapacitors with high energy densities. Adv Energy Mater (4):1300816. https://doi.org/10.1002/aenm.201470017.

25. Yang J, Xie H, Chen $\mathrm{H}$ et al (2018), Cellulose nanofibril/boron nitride nanosheet composites with enhanced energy density and thermal stability by interfibrillar cross-linking through $\mathrm{Ca}^{2+}$. $\mathrm{J}$ Mater Chem A 6(4):1403-1411. https://doi.org/10.1039/c7ta08188j.

26. Yang Q, Zhang C, Shi Z et al (2018). Luminescent and transparent nanocellulose films containing europium carboxylate groups as flexible dielectric materials. ACS Appl Nano Mater (1): 4972-4979. https://doi.org/10.1021/acsanm.8b01112. 
27. Yin Y, Zhang C, Yu W, Kang G, Yang Q, Shi Z, Xiong C (2020) Transparent and flexible cellulose dielectric films with high breakdown strength and energy density. Energy Storage Mater 26:105-111. https://doi.org/10.1016/j.ensm.2019.12.034

28. Yu G, Hu L, Liu N et al (2011). Enhancing the supercapacitor performance of graphene/ $\mathrm{MnO}_{2}$ nanostructured electrodes by conductive wrapping. Nano Lett 11(10):4438-4442. https://doi.org/10.1021/nl2026635.

29. Zang X, Zhu M, Li X et al (2015). Dynamically stretchable supercapacitors based on graphene woven fabric electrodes. Nano Energy (15):83-91. https://doi.org/10.1016/j.nanoen.2015.04.010.

30. Zhan Y, Xiong C, Yang J et al (2019). Flexible cellulose nanofibril/pristine graphene nanocomposite films with high electrical conductivity. Composites Part A (119):119-126. https://doi.org/10.1016/j.compositesa.2019.01.029.

31. Zhao L, Fan LZ, Zhou MQ et al (2010). Nitrogen-containing hydrothermal carbons with superior performance in supercapacitors. Adv Mater (22):5202-5206. https://doi.org/10.1002/adma.201002647.

\section{Figures}

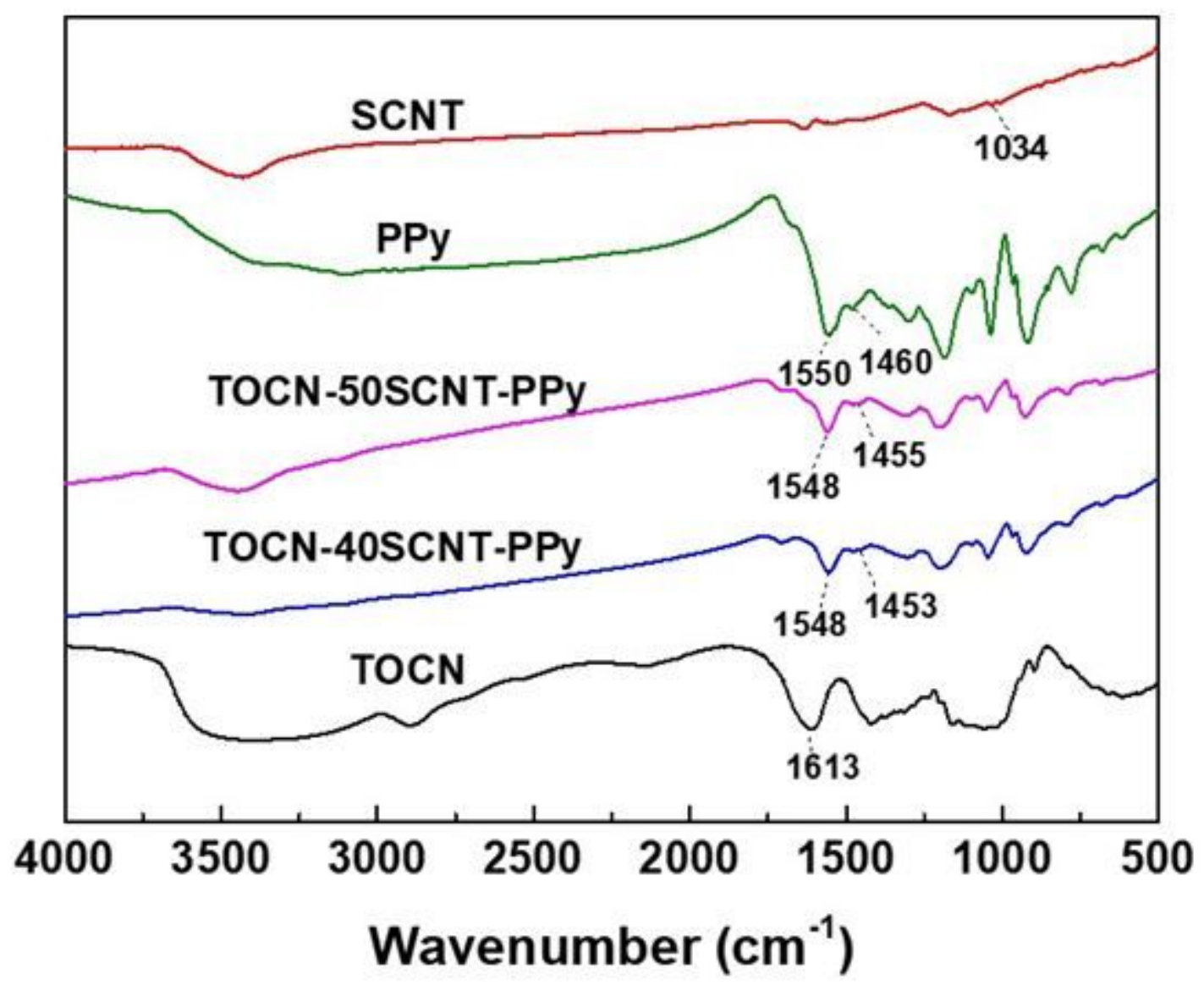


Figure 1

FT-IR spectra of TOCN, SCNT, PPy and TOCN/SCNT/PPy aerogels.

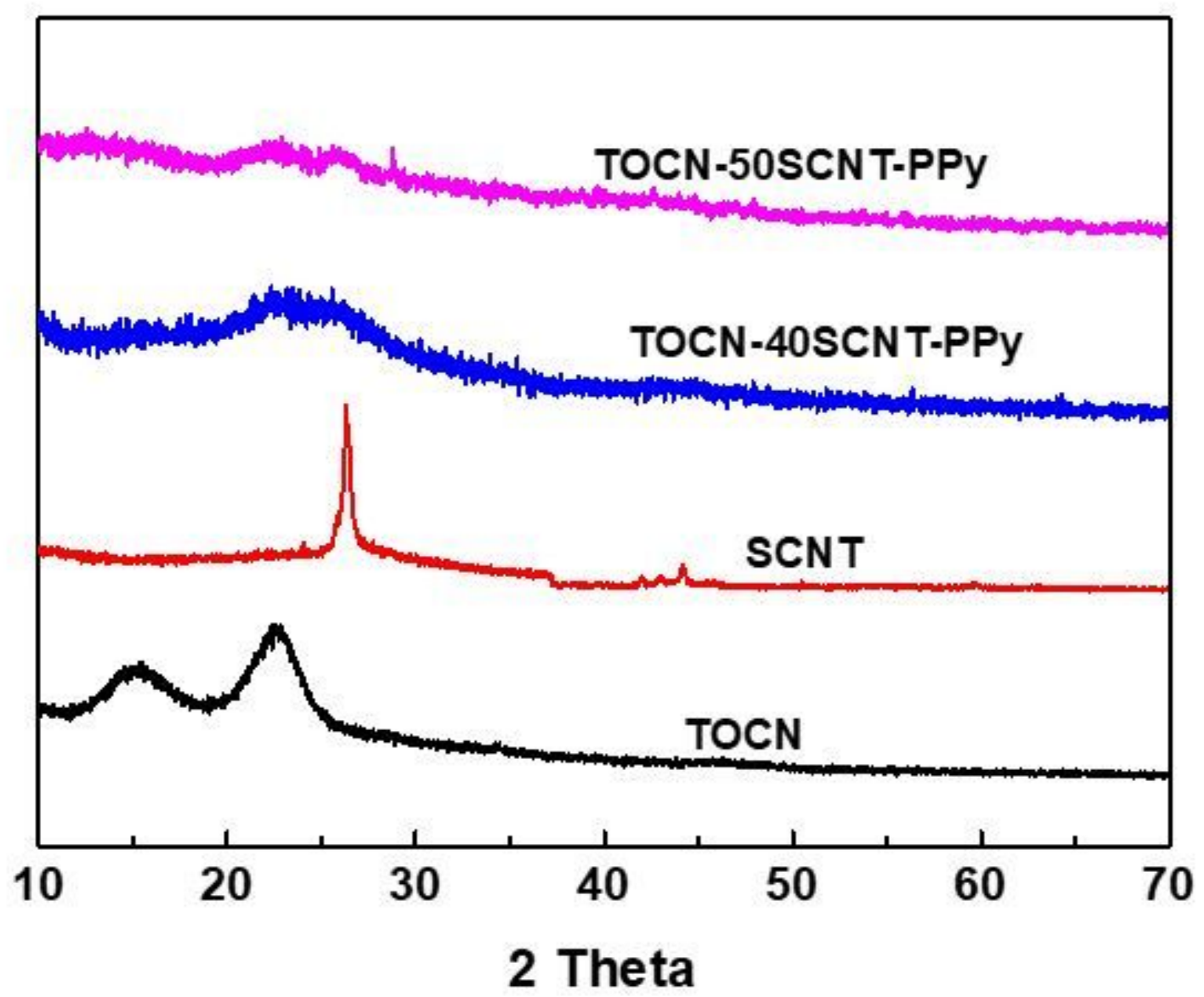

Figure 2

XRD patterns of TOCN, SCNT and TOCN/SCNT/PPy aerogels.
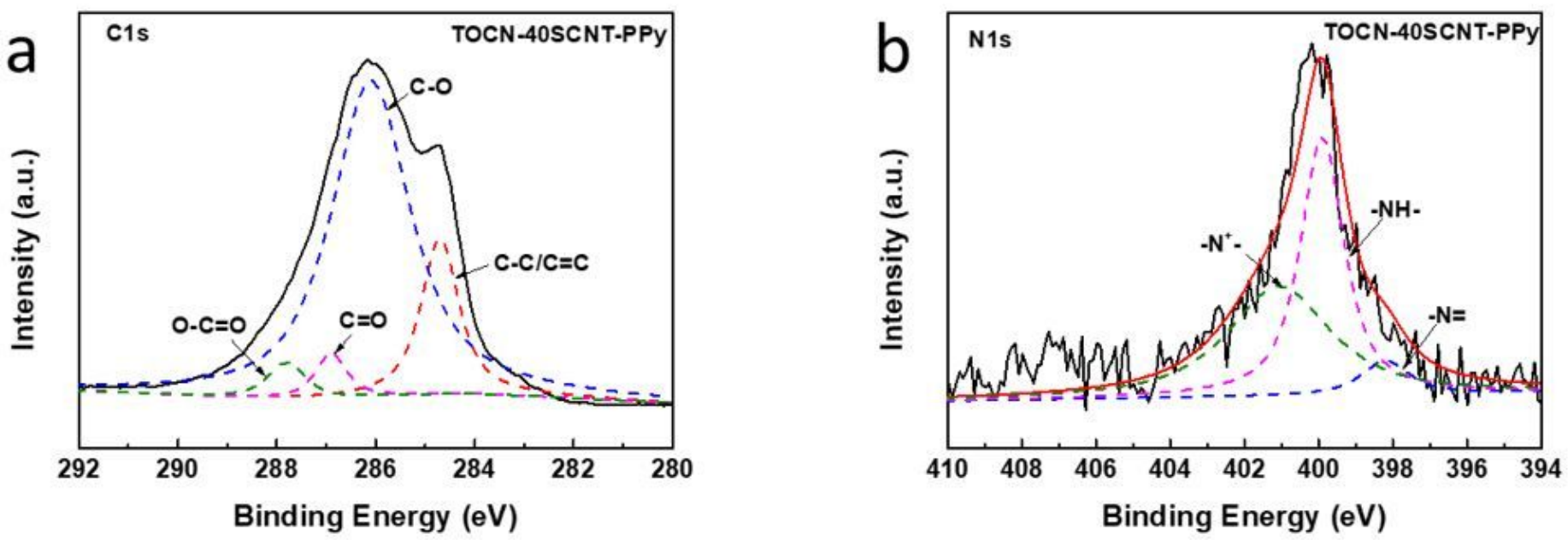

Figure 3 
XPS (a) C1s and (b) N1s spectra of the TOCN-40SCNT-PPy aerogel.
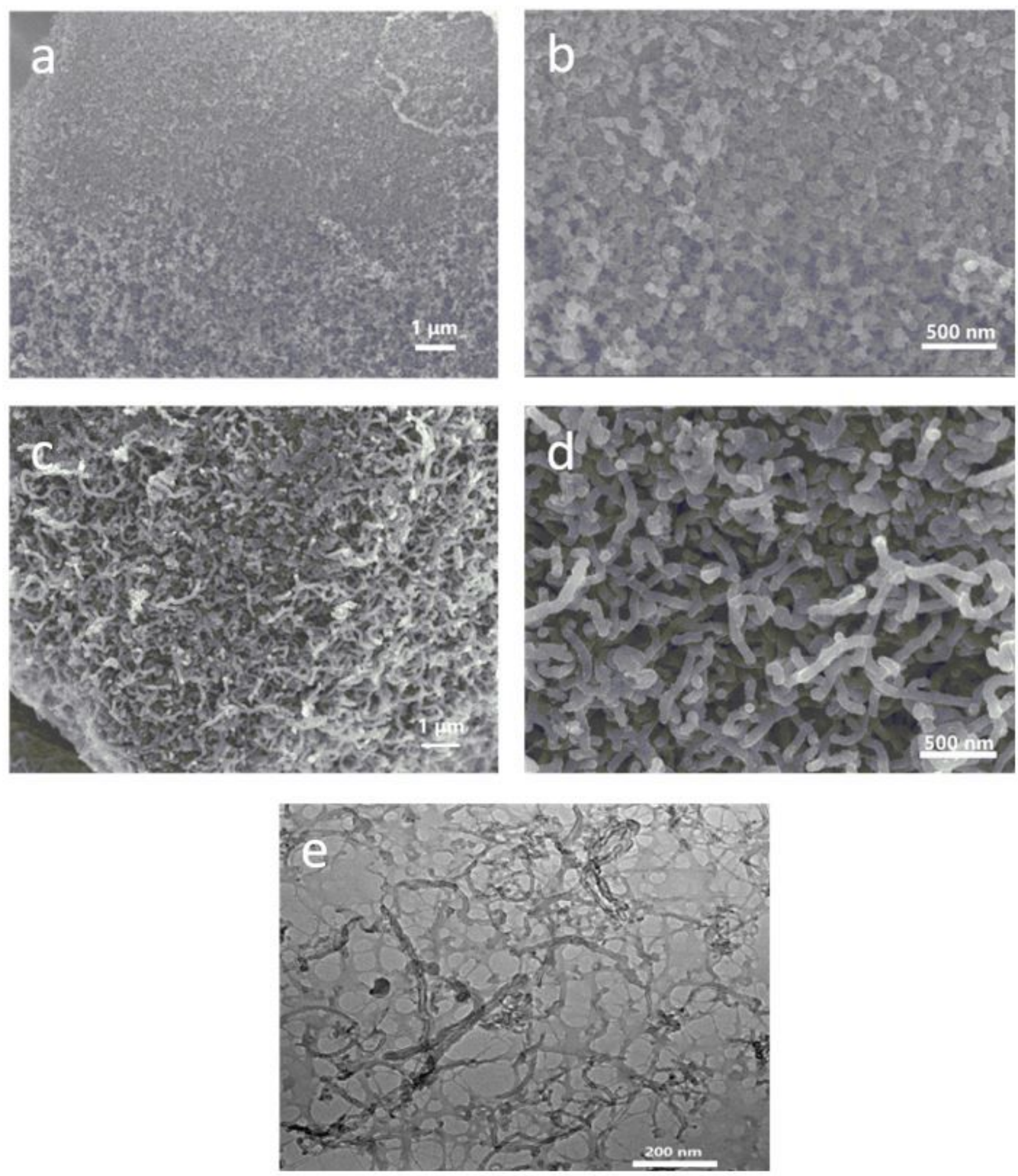

\section{Figure 4}

SEM images of $(a, b)$ TOCN-40SCNT-PPy and (c, d) TOCN-50SCNT-PPy aerogels, respectively; TEM image of (e) the TOCN-40SCNT-PPy aerogel. 

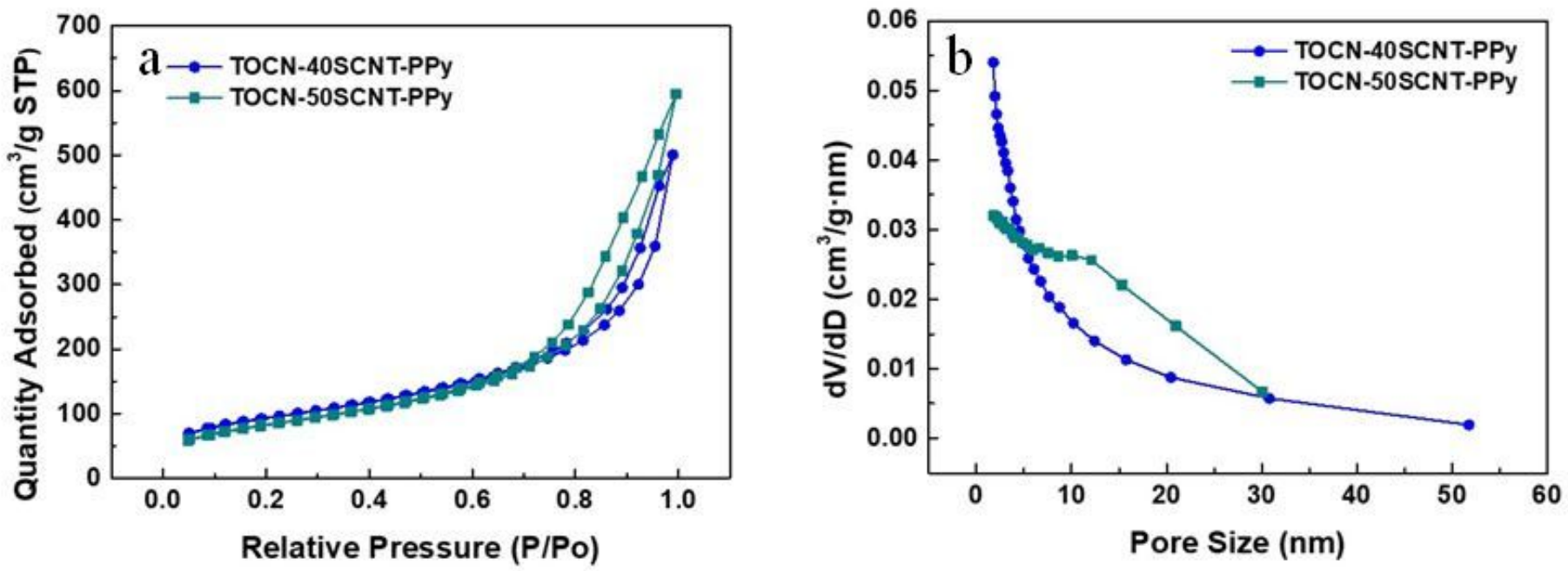

Figure 5

(a) Nitrogen adsorption/desorption isotherms and (b) Barrett-Joyner-Halenda (BJH) pore size distribution of TOCN-40SCNT-PPy and TOCN-50SCNT-PPy aerogels.
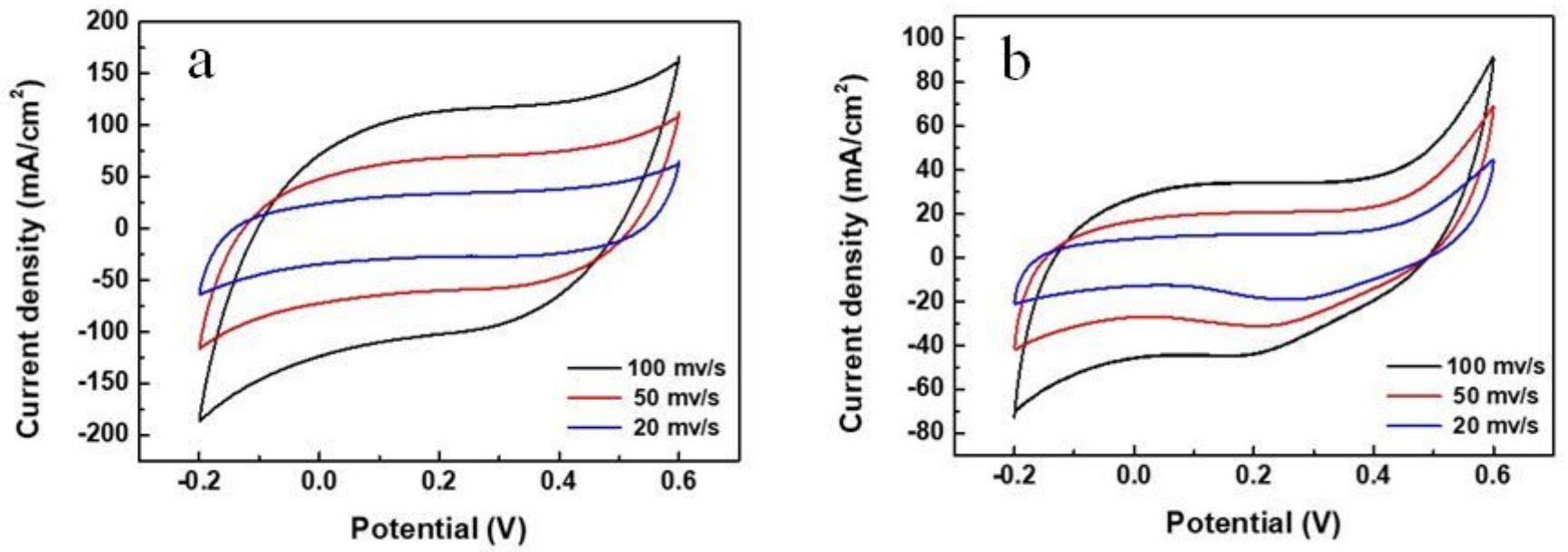

Figure 6

CV curves of (a) TOCN-40SCNT-PPy and (b) TOCN-50SCNT-PPy hydrogel electrodes. 

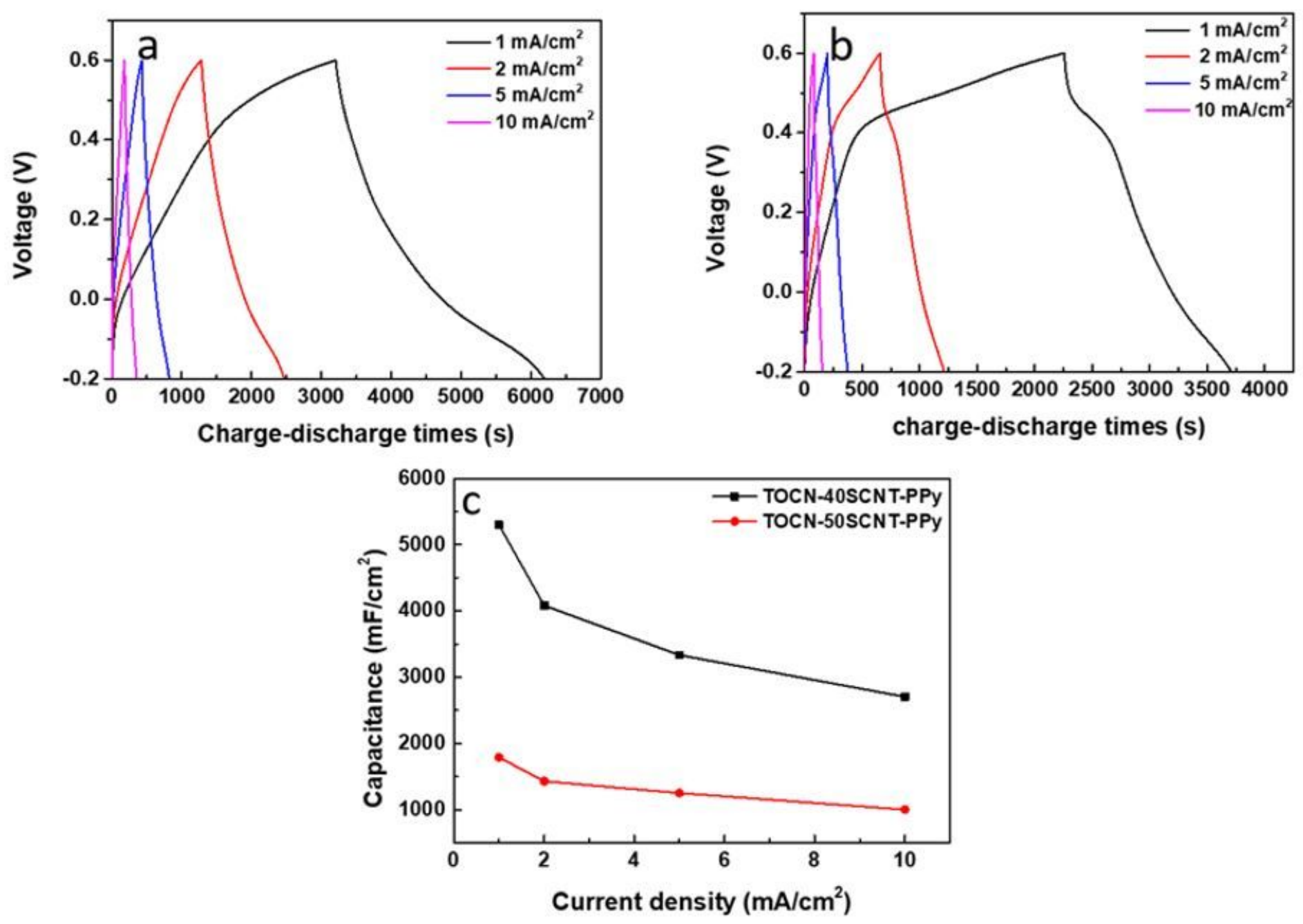

Figure 7

Galvanostatic charge-discharge (GCD) curves of (a) TOCN-40SCNT-PPy and (b) TOCN-50SCNT-PPy at different current densities. (c) Specific capacity of TOCN/SCNT/PPy samples as a function of current density ranging from $1 \mathrm{~mA} / \mathrm{cm} 2$ to $10 \mathrm{~mA} / \mathrm{cm} 2$. 


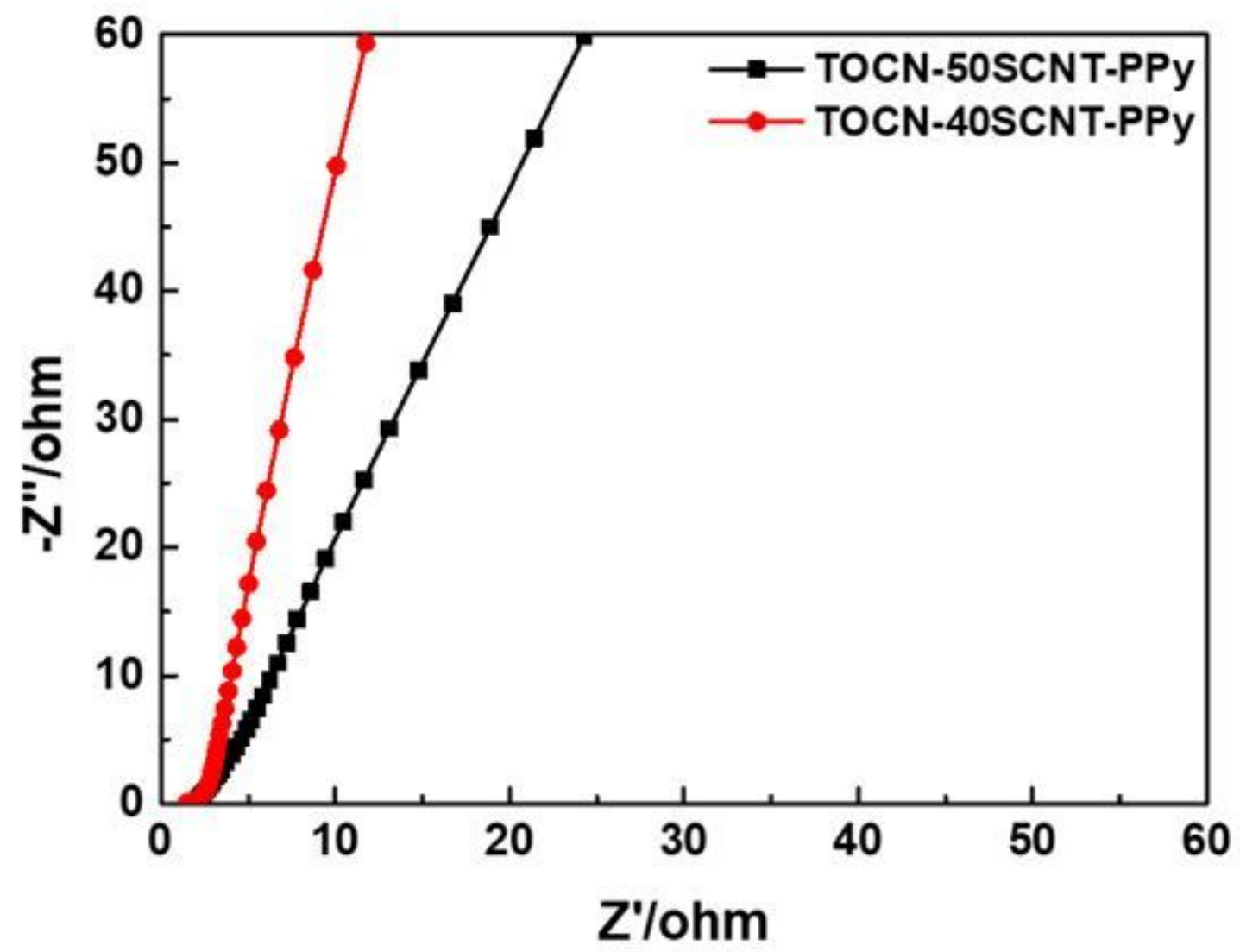

Figure 8

Nyquist impedance spectra of TOCN-40SCNT-PPy and TOCN-50SCNT-PPy hydrogels. 


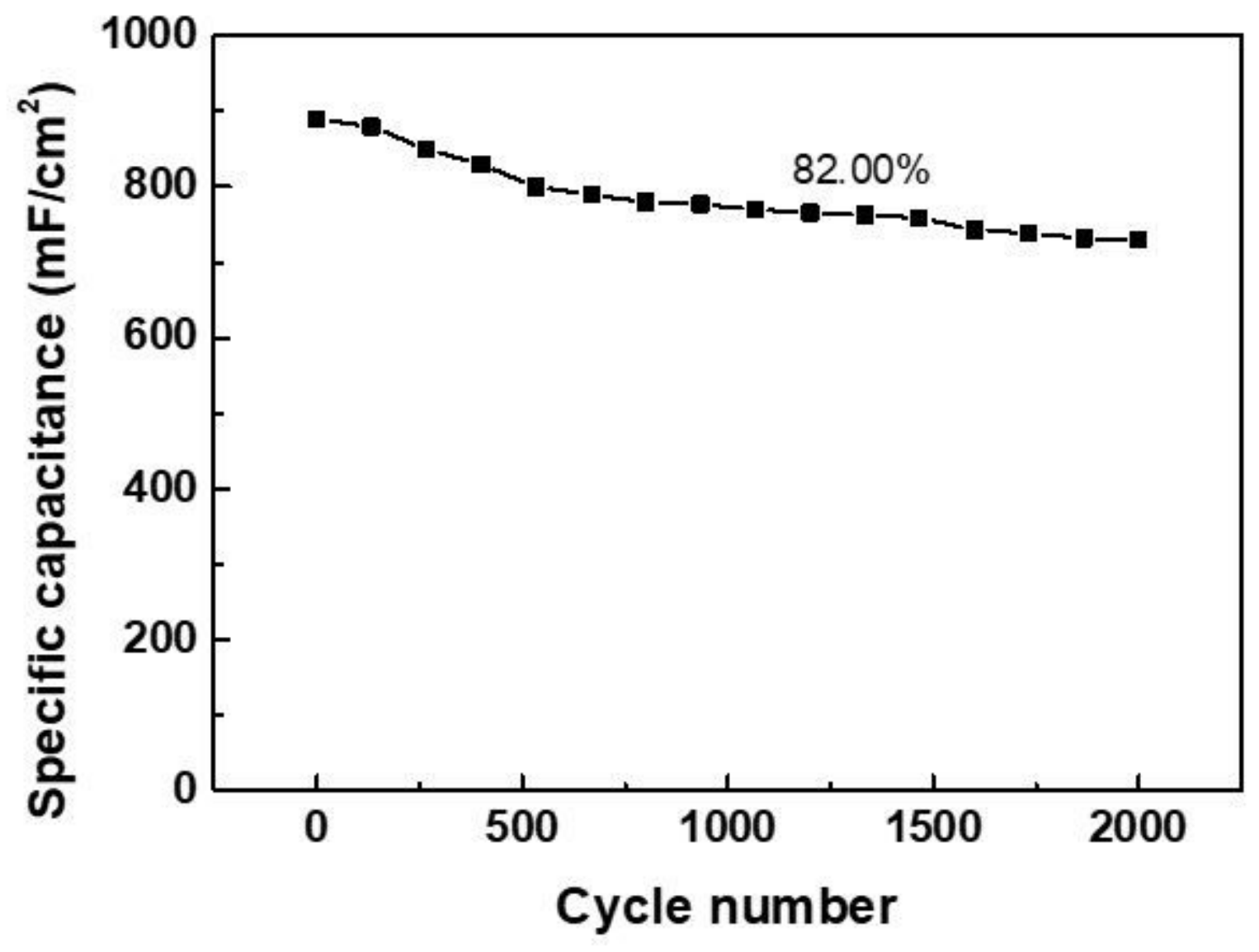

Figure 9

Cycling stability of TOCN-40SCNT-PPy over 2000 cycles at a current density of $5 \mathrm{~mA} / \mathrm{cm} 2$. 

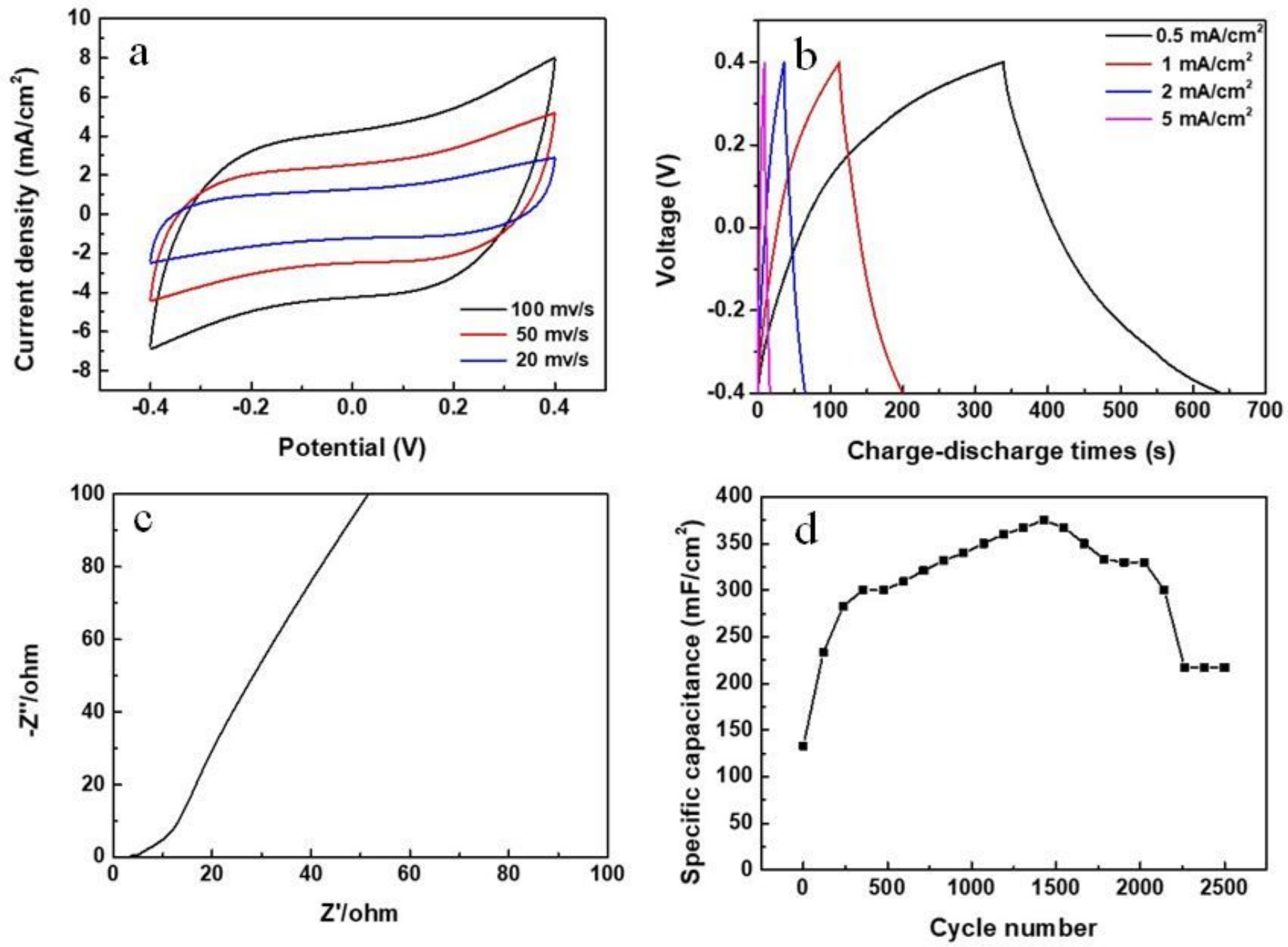

Figure 10

Two-electrode tests of a symmetric TOCN-40SCNT-PPy solid-state supercapacitor. Cyclic voltammetry (CV) curves at different scan rates (a), Galvanostatic charge-discharge curves at different current densities (b), Nyquist impedance spectra (c) and cycling stability (d) over 2500 cycles at a current density of $1 \mathrm{~mA} / \mathrm{cm} 2$.

\section{Supplementary Files}

This is a list of supplementary files associated with this preprint. Click to download.

- GraphicalAbstract.jpg

- R4TOCNSCNTPPysupportinginformation.docx 